\title{
Excess length of stay and mortality due to Clostridium difficile infection: a multi-state modelling approach
}

\author{
E. van Kleef ${ }^{a, b, *}$, N. Green ${ }^{\text {b, c }}$, S.D. Goldenberg ${ }^{\text {d }}$, J.V. Robotham ${ }^{b}$, B. Cookson ${ }^{e}$, \\ M. Jit ${ }^{a, b}$, W.J. Edmunds ${ }^{a}$, S.R. Deeny ${ }^{b}$
}

a London School of Hygiene and Tropical Medicine, London, UK

b Public Health England, Colindale, London, UK

'Imperial College London, London, UK

'Guy's and St Thomas' NHS Foundation Trust and King's College, London, UK

e University College London, London, UK

\section{A R T I C L E I N F O}

\section{Article history:}

Received 19 March 2014

Accepted 18 August 2014

Available online 18 September

2014

\section{Keywords:}

Clostridium difficile infection Length of hospital stay

Mortality

CrossMark
S U M M A R Y

Background: The burden of healthcare-associated infections, such as healthcare-acquired Clostridium difficile (HA-CDI), can be expressed in terms of additional length of stay (LOS) and mortality. However, previous estimates have varied widely. Although some have considered time of infection onset (time-dependent bias), none considered the impact of severity of HA-CDI; this was the primary aim of this study.

Methods: The daily risk of in-hospital death or discharge was modelled using a Cox proportional hazards model, fitted to data on patients discharged in 2012 from a large English teaching hospital. We treated HA-CDI status as a time-dependent variable and adjusted for confounders. In addition, a multi-state model was developed to provide a clinically intuitive metric of delayed discharge associated with non-severe and severe HA-CDI respectively.

Findings: Data comprised 157 (including 48 severe) HA-CDI cases among 42,618 patients. $\mathrm{HA}-\mathrm{CDI}$ reduced the daily discharge rate by nearly one-quarter [hazard ratio (HR): 0.72 ; 95\% confidence interval $(\mathrm{Cl}): 0.61-0.84]$ and increased the in-hospital death rate by $75 \%$ compared with non-HA-CDI patients (HR: 1.75 ; $95 \% \mathrm{Cl}: 1.16-2.62$ ). Whereas overall HA-CDI resulted in a mean excess LOS of about seven days ( $95 \% \mathrm{Cl}: 3.5-10.9)$, severe cases had an average excess LOS which was twice $(\sim 11.6$ days; $95 \% \mathrm{Cl}: 3.6-19.6)$ that of the nonsevere cases (about five days; $95 \% \mathrm{Cl}: 1.1-9.5$ ).

Conclusion: HA-CDI contributes to patients' expected LOS and risk of mortality. However, when quantifying the health and economic burden of hospital-onset of HA-CDI, the heterogeneity in the impact of HA-CDI should be accounted for.

(C) 2014 The Healthcare Infection Society. Published by Elsevier Ltd. All rights reserved.
* Corresponding author. Address: London School of Hygiene and Tropical Medicine, Keppel Street, London WC1E 7HT, UK. Tel.: +44 (0) 7858033771 .

E-mail address: esther.vankleef@lshtm.ac.uk (E. van Kleef).

\section{Introduction}

Clostridium difficile is a considerable cause of healthcareassociated infections in Europe and the USA. ${ }^{1,2}$ In common with other healthcare-associated infections, patients with healthcare-associated Clostridium difficile infection (HA-CDI) 
place a serious health and economic burden on the hospital system. Previous economic analyses of HA-CDI have shown that direct healthcare, and opportunity, costs due to excess length of hospital stay (LOS) were the main HA-CDI cost drivers. ${ }^{3-6}$ However, a recent review of publications on HA-CDI-associated additional hospital stay showed wide variation in excess LOS, ranging from 2.8 to 16.1 days. $^{7}$ These studies primarily used simple regression models, which did not account for the timing of onset of infection ('time-dependent bias'). ${ }^{8}$ Hence, they may overestimate the duration of excess hospitalization, as a longer stay in hospital may increase the risk of infection. This has been demonstrated rigorously for other healthcare-associated infections but has rarely been explicitly explored for HA-CDI. ${ }^{7-10}$

Two recent publications, which implicitly adjusted for timedependent bias, reported very different results. ${ }^{11,12}$ Using a Cox proportional hazards model, Forster et al. concluded that HA-CDI patients had a median excess LOS of six days, whereas Mitchell et al. found no significant impact of HA-CDI on hospital stay. ${ }^{11,12}$ Mitchell et al. used multi-state modelling (MSM), which - in addition to appropriately adjusting for both time to event bias and the competing end-points related to nosocomial infections, namely discharge and death - also provided more easily interpretable results than the proportional hazards model. ${ }^{11}$ In their conclusion, Mitchell et al. posit that their results, and the difference from earlier estimates, could potentially be explained by milder HA-CDI due to a lack of circulating hypervirulent polymerase chain reaction (PCR) ribotype 027 in their locality (Tasmania, Australia).

As the clinical presentation of HA-CDI can range from mild diarrhoea to pseudomembranous colitis and even death, and the prevalence of severe HA-CDI can vary regionally, due to differences in ribotype prevalence and case-mix, it is important for any estimation of attributable LOS and mortality to consider these heterogeneities. ${ }^{13-16}$ In this study, we investigate the effect of severe infection on the expected delayed discharge and mortality associated with HA-CDI based on a Cox proportional hazards model as well as MSM techniques.

\section{Methods}

\section{Data}

Data were collected from Guy's and St Thomas' National Health Service (NHS) Foundation Trust, a teaching hospital including two sites with $\sim 1200$ beds. Details of all inpatients discharged in 2012 (namely: age, primary diagnosis code, dates of admission and discharge, and discharge status; i.e. discharge alive or death) were extracted from the Trust's electronic patient record database. These data were linked to the Trust's voluntarily collected HA-CDI surveillance database containing information on date of onset of symptoms, markers of HA-CDI severity, antibiotic treatment, and, where known, the ribotype that caused the infection. In order to evaluate only those infections that were hospital-acquired, patients with symptom onset $\leq \mathbf{4 8} \mathrm{h}$ after admission and all patients with LOS $\leq 48 \mathrm{~h}$ were excluded from analyses.

\section{Severe $H A-C D I$}

Severe HA-CDI was defined by the clinical presence of one or more of four indicators: (i) peripheral white blood cell count
$>15 \times 10^{9} / \mathrm{L}$; (ii) acutely rising serum creatinine (>50\% increase above baseline); (iii) temperature $>38.5^{\circ} \mathrm{C}$; or (iv) radiographic evidence of colitis or endoscopic appearance of pseudomembranous colitis. ${ }^{17}$

\section{Procedure}

Proportions and medians were compared using the $\chi^{2}$-test and Mann-Whitney-Wilcoxon test, respectively. A Cox proportional hazards model was used to estimate the impact of HA-CDI on the risk of hospital discharge alive or death. This method can adjust for time-dependent bias and take into account the impact of important non-healthcare-associated infection variables such as age and comorbidity score on LOS. Hence, the method can identify and adjust for important confounders. Nonetheless, it does not produce an easily interpretable metric (namely hazard ratios) for economic analysis. Therefore we constructed a suitable MSM, to quantify the average excess LOS caused by the event of interest, i.e. HA-CDI. However, using established methodology, MSM does not account for the potential confounding effect of other variables. As an alternative, we stratified our data by each of the relevant confounders, and performed the MSM on these subsamples.

\section{Statistical analysis}

\section{Cox regression model}

The risk of in-hospital death or discharge was modelled with a Cox proportional hazards model, with HA-CDI treated as a time-dependent risk factor. We added the covariates age and comorbidity to assess their confounding effects on the risk of in-hospital death or discharge. Comorbidity was expressed in a Charlson Comorbidity Index $(\mathrm{CCl})$ score based on the patients' primary diagnosis code. ${ }^{18}$ These primary diagnoses were classified using International Statistical Classification of Diseases and Related Health Problems, 10th Revision (ICD-10) codes. ${ }^{18}$

\section{Multi-state model}

The first MSM comprised four states; admission with no HACDI (state 0), HA-CDI (state 1), discharge alive (state 2), and death in hospital (state 3$).{ }^{19}$ Since we were solely concerned with HA-CDI, it was assumed that all patients were admitted to the hospital without infection. Uninfected patients remained in state 0 from admission until discharge (state 2 ) or in-hospital death (state 3 ). Infected patients entered state 1 and then remained in that state until discharge or death. This competing end-points approach allowed for assessment of the impact of mortality due to HA-CDI on patients' expected excess stay, e.g. whether HA-CDI-related mortality may shorten expected LOS. Second, prolonged LOS associated with HA-CDI was estimated by constructing an MSM in which the two competing end-points were combined as a single state. ${ }^{20}$ Transitions between states were determined by time-varying hazards, which were estimated using the Aalen-Johansen estimator. ${ }^{21}$ For each point in time $t$ (in days), the expected LOS for HA-CDI and non-infected patients was compared. The unadjusted expected change in LOS was then calculated as the average difference in LOS of $\mathrm{HA}-\mathrm{CDI}$ and non-HA-CDI cases across all days, weighted relative to the frequency of the possible events (i.e. HA-CDI, discharge alive and in-hospital death) on each day. ${ }^{22}$ Bootstrapping was 
used to obtain robust standard error-based confidence intervals. $^{23}$

To assess the effect of specific confounders identified by the Cox regression model, the MSM compared patients within their risk group by stratification, for each potential confounding variable separately. To evaluate the effect of severe HA-CDI on excess LOS, a stratified analysis was conducted, comparing mean differences in LOS of non-severe HA-CDI and severe HA$\mathrm{CDI}$ cases respectively to non-infected patients.

All analyses were performed with R 3.0.1 (Team R Development Core: http://cran.r-project.org/). The R-packages mvna and etm were used to estimate the excess LOS and standard errors, and the R-package 'survival' was used for the Cox model. ${ }^{21,24}$

\section{Results}

\section{Descriptive statistics}

The data comprised a total sample of 42,618 patients; 157 (0.4\%) had an episode of HA-CDI, of which 48 (30.6\%) were severe (Table I). The median age of the infected patients was 72 years [interquartile range (IQR): 57-82], which was significantly higher than the median age of non-infected patients (47 years; IQR: $26-68 ; P<0.0001)$. The vast majority of all patients had no reported comorbidities (91.6\%, 39,146 patients). This percentage was slightly lower in the HA-CDI-infected patient group $(84.7 \%, 133$ patients) than in the non-infected group $(91.9 \%, 39,013$ patients; $P=0.002)$. On average, patients with severe HA-CDI had spent a shorter time in hospital until identification of the infection than non-severe cases (Table I). Moreover, after detection, the median LOS was longer for severely infected patients (Table I). Finally, the causative PCR ribotypes were known for 113 (72.0\%) of the HA$\mathrm{CDI}$ patients. None of these patients had an infection caused by hypervirulent PCR ribotype 027.

\section{Cox regression model results}

HA-CDI-positive patients had a lower daily chance of being discharged (alive or dead) than non-infected patients (Table II). Both age of the patient (i.e. $>65$ or $<65$ years), and the patient's comorbidity were significant confounders, and the adjusted daily hazard of discharge (hazard ratio: HR) for
HA-CDI patients was $0.72[95 \%$ confidence interval $(\mathrm{Cl})$ : 0.61-0.84] (Table II). Moreover, HA-CDI patients were at higher risk of experiencing in-hospital death than HA-CDI-negative patients (HR: 1.75; 95\% Cl: 1.16-2.62). Accounting for severity of the infection revealed that both non-severe and severe cases had a significantly reduced daily likelihood of discharge compared to non-infected cases, with a further reduction for the severe CDI group (Table II, Figure 1). However, severe infection did not result in a further elevated chance of dying in hospital (Table II).

\section{Multi-state model results}

The four-state multi-state model, which allowed for competing end-points, and the three-state combined end-point model yielded similar estimates for the excess LOS. Thus mortality due to HA-CDI did not seem to have an impact on the estimated additional days of hospitalization associated with HA$\mathrm{CDI}$. For this reason, only the outcomes of the combined endpoint model are presented. The average extra number of days (unadjusted for confounders) spent in hospital due to HA-CDI was about seven days (95\% $\mathrm{Cl}$ : 4-11) (Table II). Patients with severe HA-CDI had, on average, twice the additional LOS of non-severe cases, but with overlapping confidence intervals (Table II). Stratification of our sample by age and comorbidity score still showed an impact of HA-CDI on the patients' stay. Among HA-CDI patients aged $<65$ years ( 55 in total), there was an average excess LOS of about seven days ( $95 \% \mathrm{Cl}: 1-14)$ associated with $\mathrm{HA}-\mathrm{CDI}$; for patients aged $>65$ years (133 in total) this was about six days (95\% $\mathrm{Cl}: 2-11)$. A sample restricted to patients with a comorbidity score $<1$ (133 patients) showed an excess LOS of about seven days (95\% $\mathrm{Cl}$ : $3-11)$ whereas an average excess LOS of $\sim 10$ days ( $95 \% \mathrm{Cl}:-1$ to 21 ) associated with HA-CDI was found for patients with a score of $>1$ ( 24 patients).

\section{Discussion}

This study showed that HA-CDI resulted in a prolonged LOS of about seven days. Moreover, the daily mortality rate of HA-CDI patients was almost twice that of non-HA-CDI-infected patients, as has been suggested elsewhere. ${ }^{26}$ Severe infection increased the average expected excess LOS associated with HACDI. Our LOS estimates are comparable to the median additional six days estimated by a Canadian study, which also adjusted for

Table I

Demographic characteristics of the Guy's and St Thomas' hospital patients discharged in 2012

\begin{tabular}{|c|c|c|c|c|c|}
\hline Variable & $\begin{array}{l}\text { Non-infected } \\
(N=42,461)\end{array}$ & Infected $(N=157)$ & $\begin{array}{l}\text { Non-severe HA-CDI } \\
\quad(N=109)\end{array}$ & $\begin{array}{l}\text { Severe HA-CDI } \\
\quad(N=48)\end{array}$ & $\begin{array}{c}\text { Total } \\
(N=42,618)\end{array}$ \\
\hline $\begin{array}{l}\text { Age }>65 \text { years } \\
\mathrm{CCl} \text { score }\end{array}$ & $13,446(31.7 \%)$ & $102(65.0 \%)$ & $73(67.0 \%)$ & $29(60.4 \%)$ & $13,548(31.8 \%)$ \\
\hline 0 & $39,013(91.9 \%)$ & $133(84.7 \%)$ & $96(88.1 \%)$ & 37 (77.1\%) & $39,146(91.9 \%)$ \\
\hline$\geq 1$ & $3448(8.1 \%)$ & $24(15.3 \%)$ & $13(11.9 \%)$ & $11(22.9 \%)$ & 3472 (8.1\%) \\
\hline Death & $801(1.9 \%)$ & $24(15.3 \%)$ & $15(13.8 \%)$ & 9 (18.7\%) & $825(1.9 \%)$ \\
\hline Length of stay ${ }^{a}$ & $4.0 / 7.6(2.0-8.0)$ & $25.0 / 36.6(15.0-50.0)$ & $29.0 / 36.6(15.0-50.0)$ & $22.5 / 36.8(14.8-44.8)$ & $4.0 / 7.7(2.0-8.0)$ \\
\hline $\begin{array}{l}\text { Length of stay } \\
\text { pre infection }\end{array}$ & NA & $9.0 / 15.8(4.0-18.0)$ & $10.0 / 17.1(4.0-22.0)$ & $7.0 / 12.1(3.8-12.3)$ & NA \\
\hline $\begin{array}{l}\text { Length of stay } \\
\text { post infection }\end{array}$ & NA & $12.0 / 21.1(6.0-25.0)$ & $11.0 / 19.5(6.0-24.0)$ & $13.5 / 24.6(7.8-27.8)$ & NA \\
\hline
\end{tabular}

HA-CDI, healthcare-associated Clostridium difficile infection; $\mathrm{CCI}$, Charlson Comorbidity Index; NA, not applicable.

${ }^{a}$ Median/mean (interquartile range). 
Table II

Hazard ratios for in-hospital death or discharge alive and excess length of stay estimated from the Cox regression model and multi-state model

\begin{tabular}{|c|c|c|c|c|c|c|c|}
\hline \multirow[t]{2}{*}{ Exposure } & \multicolumn{2}{|c|}{$\begin{array}{l}\text { Hazard ratio }(95 \% \mathrm{Cl}) \text { for } \\
\text { discharge alive }\end{array}$} & \multicolumn{2}{|c|}{$\begin{array}{c}\text { Hazard ratio }(95 \% \mathrm{Cl}) \text { for } \\
\text { in-hospital death }^{a}\end{array}$} & \multicolumn{2}{|c|}{$\begin{array}{l}\text { Hazard ratio }(95 \% \mathrm{Cl}) \text { for } \\
\text { discharge alive or death }\end{array}$} & \multirow[t]{2}{*}{$\begin{array}{l}\text { Excess LOS } \\
(95 \% \mathrm{Cl})^{d}\end{array}$} \\
\hline & Time-adjusted $^{\mathrm{b}}$ & Fully adjusted $^{c}$ & Time-adjusted $^{\mathrm{b}}$ & Fully adjusted $^{c}$ & Time-adjusted $^{\mathrm{b}}$ & Fully adjusted $^{c}$ & \\
\hline All CDI & $0.66(0.56-0.77)$ & $0.72(0.61-0.84)$ & $1.98(1.33-2.96)$ & $1.75(1.16-2.62)$ & $0.73(0.64-0.84)$ & $0.79(0.69-0.92)$ & $7.2(3.5-10.9)$ \\
\hline Severe CDI & $0.53(0.39-0.71)$ & $0.59(0.44-0.79)$ & $2.11(1.17-3.79)$ & $1.76(0.95-3.25)$ & $0.62(0.49-0.76)$ & $0.69(0.54-0.85)$ & $11.6(3.6-19.6)$ \\
\hline $\begin{array}{l}\text { Non-severe } \\
\text { CDI }\end{array}$ & $0.73(0.60-0.88)$ & $0.79(0.66-0.96)$ & $1.91(1.13-3.22)$ & $1.74(1.03-2.93)$ & $0.80(0.67-0.95)$ & $0.86(0.71-1.03)$ & $5.3(1.1-9.5)$ \\
\hline
\end{tabular}

$\mathrm{CI}$, confidence interval; LOS, length of hospital stay; CDI, Clostridium difficile infection.

To estimate the hazard ratios for discharge alive and in-hospital death respectively, the observations on patients who experienced a competing risk event were censored, i.e. removed from the risk set used for the hazard calculation at time of occurrence of the event, based on the principles of the cause-specific hazard function. ${ }^{25}$

${ }^{\text {a }}$ Cox regression model.

${ }^{b}$ Cox regression model with time to infection included as a time-dependent variable.

c Cox regression model with time to infection included as a time-dependent variable, and age ( $<65$ and $>65$ years) and comorbidity (Charlson Comorbidity Index score $<1$ and $\geq 1$ ) included as covariates.

d Multi-state model.

the time dependency of the impact of HA-CDI, but contradict the findings of a recent study by Mitchell et al. in an Australian hospital using a comparable MSM, suggesting that HA-CDI did not result in excess LOS. ${ }^{7,12}$ As the MSM of Mitchell et al. did not implicitly adjust for such potential confounders, difference in case-mix among the different hospital settings (UK and Australia) could have been responsible for the differences in measured excess LOS. ${ }^{27}$ However, stratified MSM results (by age, and comorbidity index score respectively) revealed that both younger and older HA-CDI patients had an increased average LOS of about six or seven days, and patients of both comorbidity groups had an increased expected LOS related to CDI, so this is unlikely to supply the explanation. Moreover, the non-severe HA-CDI patients in our sample still had a significantly increased LOS of about five days; thus, even the potential lack of severely infected patients in the Australian sample would have most likely resulted in an effect on LOS. However, with only three published studies using appropriate methods, little can be concluded with regard to the heterogeneity in the findings. More appropriate analyses of existing datasets (e.g. those reviewed by Mitchell et al.) might be a sensible way forward. ${ }^{7}$

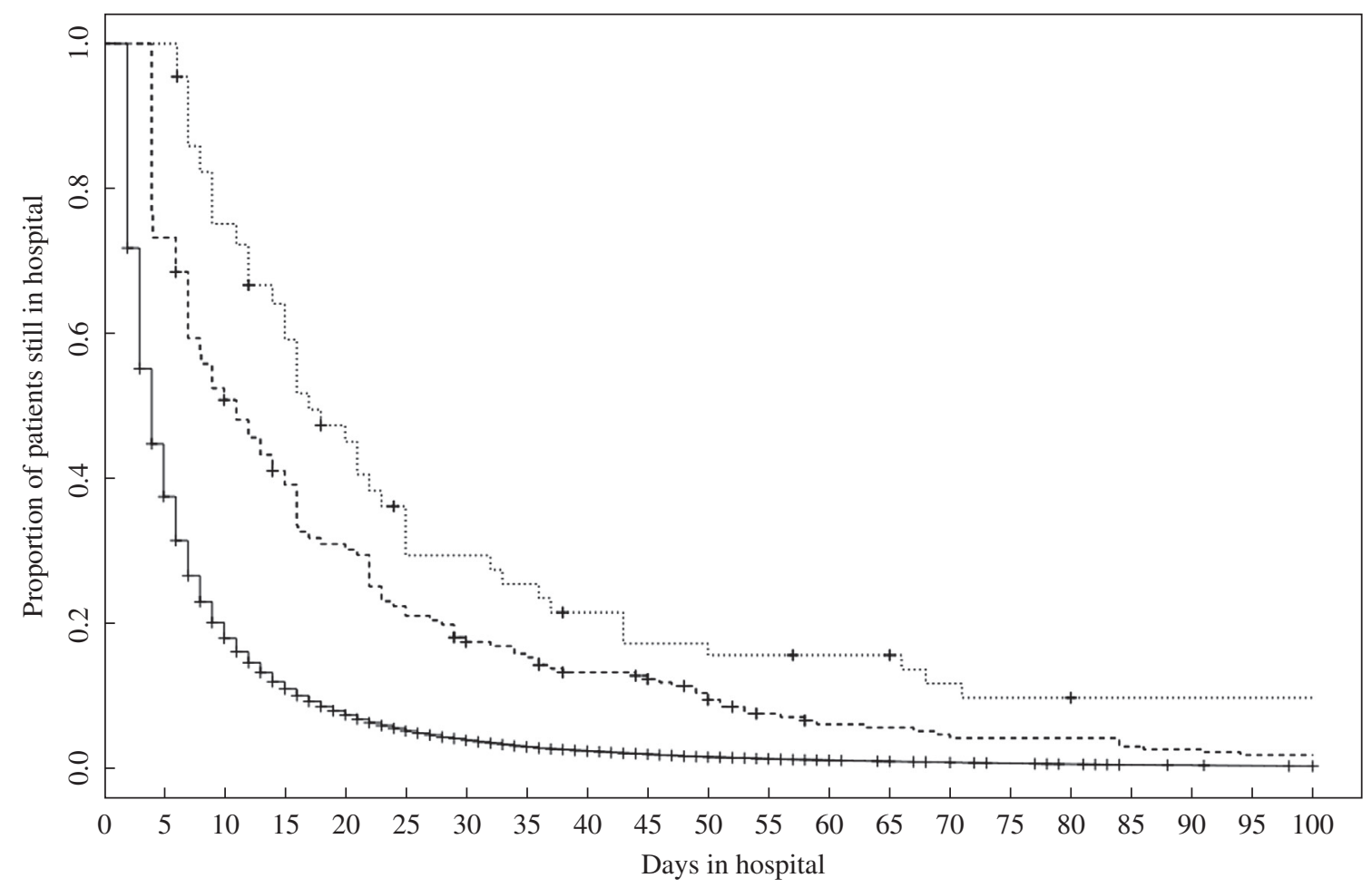

Figure 1. Kaplan-Meier curve for the time-adjusted length of hospital stay for patients without healthcare-associated Clostridium difficile infection (HA-CDI) (solid line), non-severe HA-CDI (dashed line), and severe HA-CDI (dotted line). + denote censored observations (i.e. death). 
Our dataset was collected as part of routine record keeping in a large teaching hospital, which led to a number of limitations in our study. First, the number of patients with HA-CDI was small, resulting in relatively large standard errors for the risk-group-stratified MSM analysis. A larger dataset would have allowed sufficient power to sub-stratify the analysis further. Second, the comorbidity index was calculated using only the primary diagnosis code of each patient, as more detailed information was not available to the researchers. Therefore, our analysis might not have fully adjusted for the effect of concomitant conditions. Finally, patients who spend a longer time in hospital are at increased risk of acquiring healthcareassociated infections other than HA-CDI, which could have extended their LOS rather than HA-CDI. However, considering the construction of the MSM, this may be explained as follows: HA-CDI-positive patients who were in hospital for a given number of days post onset were compared to admitted HA-CDInegative patients still in hospital after this number of days. Thus, both these populations were arguably equally at risk of acquiring healthcare-associated infections other than HA-CDI.

In conclusion, the present work confirms the heterogeneity among patients concerning the health and economic burden of CDI. To our knowledge, these results have yielded the first severity-specific estimate of the additional LOS and excess mortality due to CDI. We believe that the techniques presented here could supply policy-makers - locally, nationally, and regionally - with an estimate of the burden of CDI for their patient population and severity of CDI.

\section{Conflict of interest statement None declared.}

\section{Funding sources}

This work was supported by the Healthcare Infection Society Grant: Modelling the population-level effect and cost-effectiveness of $C$. difficile vaccination as part of an integrated healthcare and associated infection prevention and control strategy.

\section{References}

1. Bauer MP, Notermans DW, van Benthem BHB, et al. Clostridium difficile infection in Europe: a hospital-based survey. Lancet 2011;377(9759):63-73.

2. Kuijper EJ, Coignard B, Tüll P. Emergence of Clostridium difficile-associated disease in North America and Europe. In: Kuijper EJ, Coignard B, Tull P, editors. Clin Microbiol Infect 2006;12. 2-18.

3. Dubberke ER, Olsen MA. Burden of Clostridium difficile on the healthcare system. Clin Infect Dis 2012;55:S88-S92.

4. Dubberke ER, Reske KA, Olsen MA, McDonald LC, Fraser VJ. Shortand long-term attributable costs of Clostridium difficile-associated disease in nonsurgical inpatients. Clin Infect Dis 2008;46:497-504.

5. McGlone SM, Bailey RR, Zimmer SM, et al. The economic burden of Clostridium difficile. Clin Microbiol Infect 2012;18:282-289.

6. Ghantoji SS, Sail K, Lairson DR, DuPont HL, Garey KW. Economic healthcare costs of Clostridium difficile infection: a systematic review. J Hosp Infect 2010;74:309-318.

7. Mitchell BG, Gardner A. Prolongation of length of stay and Clostridium difficile infection: a review of the methods used to examine length of stay due to healthcare associated infections. Antimicrob Resist Infect Control 2012;1:14.
8. Van Walraven C, Davis D, Forster AJ, Wells GA. Time-dependent bias was common in survival analyses published in leading clinical journals. J Clin Epidemiol 2004;57:672-682.

9. Barnett AG, Beyersmann J, Allignol A, Rosenthal VD, Graves N, Wolkewitz M. The time-dependent bias and its effect on extra length of stay due to nosocomial infection. Value Health 2011;14:381-386.

10. Graves N, Harbarth S, Beyersmann J, Barnett A, Halton K, Cooper B. Estimating the cost of health care-associated infections: mind your p's and q's. Clin Infect Dis 2010;50:1017-1021.

11. Mitchell BG, Gardner A, Barnett AG, Hiller JE, Graves N. The prolongation of length of stay because of Clostridium difficile infection. Am J Infect Control 2014;42:164-167.

12. Forster AJ, Taljaard M, Oake N, Wilson KW, Roth V, van Walraven C. The effect of hospital-acquired infection with Clostridium difficile on length of stay in hospital. Can Med Assoc J 2012;184:17-18.

13. Wilcox MH, Shetty N, Fawley WN, et al. Changing epidemiology of Clostridium difficile infection following the introduction of a national ribotyping-based surveillance scheme in England. Clin Infect Dis 2012;55:1056-1063.

14. Walker AS, Eyre DW, Wyllie DH, et al. Relationship between bacterial strain type, host biomarkers, and mortality in Clostridium difficile infection. Clin Infect Dis 2013;56:1589-1600.

15. Goorhuis A, Bakker D, Corver J, et al. Emergence of Clostridium difficile infection due to a new hypervirulent strain, polymerase chain reaction ribotype 078. Clin Infect Dis 2008; 47:1162-1170.

16. Henrich TJ, Krakower D, Bitton A, Yokoe DS. Clinical risk factors for severe Clostridium difficile-associated disease. Emerg Infect Dis 2009; 15:415-422.

17. Debast SB, Bauer MP, Kuijper EJ. European Society of Clinical Microbiology and Infectious Diseases: update of the treatment guidance document for Clostridium difficile infection. Clin Microbiol Infect 2014;20:1-26.

18. Sundararajan V, Henderson T, Perry C, Muggivan A, Quan H, Ghali WA. New ICD-10 version of the Charlson comorbidity index predicted in-hospital mortality. J Clin Epidemiol 2004;57: 1288-1294.

19. Beyersmann J, Wolkewitz M, Allignol A, Grambauer N, Schumacher M. Application of multistate models in hospital epidemiology: advances and challenges. Biom J 2011;53: $332-350$

20. Angelis G de, Allignol A, Murthy A, et al. Multistate modelling to estimate the excess length of stay associated with meticillinresistant Staphylococcus aureus colonisation and infection in surgical patients. J Hosp Infect 2011;78:86-91.

21. Allignol A, Schumacher M, Beyersmann J. Empirical transition matrix of multi-state models: the etm package. J Stat Softw 2011;38:1-15.

22. Schulgen G, Schumacher M. Estimation of prolongation of hospital stay attributable to nosocomial infections: new approaches based on multistate models. Lifetime Data Anal 1996;2:219-240.

23. Davison AC, Hinkley DV. Bootstrap methods and their application. New York: Cambridge University Press; 1997.

24. Jackson $\mathrm{CH}$. Multistate models for panel data. J Stat Softw 2011;38(8).

25. Lau B, Cole SR, Gange SJ. Competing risk regression models for epidemiologic data. Am J Epidemiol 2009;170:244-256.

26. Hensgens MPM, Goorhuis A, Dekkers OM, van Benthem BHB, Kuijper EJ. All-cause and disease-specific mortality in hospitalized patients with Clostridium difficile infection: a multicenter cohort study. Clin Infect Dis 2013;56:1108-1116.

27. Louie TJ, Miller MA, Crook DW, Lentnek A. Effect of age on treatment outcomes in Clostridium difficile infection. J Am Geriatr Soc 2013;61:222-230. 